\title{
In defence of explanatory realism
}

\section{Stefan Roski ${ }^{1}$}

Received: 9 June 2021 / Accepted: 9 September 2021 / Published online: 17 September 2021

(c) The Author(s) 2021

\begin{abstract}
Explanatory realism is the view that explanations work by providing information about relations of productive determination such as causation or grounding. The view has gained considerable popularity in the last decades, especially in the context of metaphysical debates about non-causal explanation. What makes the view particularly attractive is that it fits nicely with the idea that not all explanations are causal whilst avoiding an implausible pluralism about explanation. Another attractive feature of the view is that it allows explanation to be a partially epistemic, context-dependent phenomenon. In spite of its attractiveness, explanatory realism has recently been subject to criticism. In particular, Taylor (Philos Stud 175(1):197-219, 2018). has presented four types of explanation that the view allegedly cannot account for. This paper defends explanatory realism against Taylor's challenges. We will show that Taylor's counterexamples are either explanations that turn out to provide information about entities standing in productive determination relations or that they are not genuine explanations in the first place.
\end{abstract}

Keywords Explanation · Metaphysics · Causation · Grounding

\section{Introduction}

Explanatory realism is the view that all explanations provide information about relations of productive determination such as, inter alia, causation and grounding. The view has gained considerable popularity in the last decades, especially in the context of metaphysical debates about non-causal explanation. ${ }^{1}$ What makes explanatory realism particularly attractive is that it fits nicely with the idea that not all explanations are causal whilst avoiding an implausible pluralism about explanation. Although explanations provide information about various different relations of productive determination, there is only one basic explanation-relation, and one basic notion of explanation pick-

1 See Schaffer (2016), Ruben (2012), Audi (2012a, b), Kim (1994, 1988).

$\bowtie \quad$ Stefan Roski

stefan.roski@gmail.com

1 Universität Hamburg, Überseering 35, Postfach \#4, 22297 Hamburg, Germany 
ing out that relation. Another attractive feature of the view is that it allows explanation to be a partially epistemic, context-dependent phenomenon. To some extent, context and epistemic background might determine whether or not some information is explanatory. But information cannot be explanatory unless it is about entities standing in productive determination relations.

In spite of its attractiveness, explanatory realism has been subject to criticism. In particular, Taylor (2018) has recently presented four types of explanations that the view allegedly cannot account for: analogical explanations, explanations by rules, explanations by reductio ad absurdum, and certain statistical explanations. Such explanations, she argues, are well established in scientific practice but do not provide information about productive determination relations. She further claims that restricting the scope of explanatory realism to merely a particular kind of explanation (e.g. to metaphysical as opposed to scientific explanation) may avoid those counterexamples but would also make the position unable to support many arguments for which it is employed in metaphysical debates; such as, for instance, arguments for features of grounding from features of explanation, or Kim's exclusion argument.

In this paper, I will defend explanatory realism against Taylor's challenges. I will show that her alleged counterexamples can in fact be accounted for by explanatory realism, properly understood. Engaging with Taylor's arguments is valuable nonetheless, as they pinpoint several features of explanatory realism that are often not spelled out in sufficient detail. This pertains, in particular, to the sense in which explanations are about entities standing in determination relations.

Here is how we shall proceed: I first introduce the general idea of explanatory realism, and identify a common denominator that most explanatory realists do, or should, agree on (Sect. 2). Subsequently, I defend the account against Taylor's criticisms. In this, I focus on her examples from analogical explanation (Sect. 3), rule-based explanation (Sect. 4), and explanation by reductio (Sect. 5). I exclude the category of statistical explanation as these cases — as Taylor admits herself — do not pose straightforward counterexamples to realism but rather prompt further developments in the theory of probabilistic causation or probabilistic grounding. ${ }^{2}$

\section{Explanatory realism}

Stating what explanatory realism amounts to is not trivial. Both proponents and critics of the account usually introduce it in a relatively informal way that is open for further elaboration. ${ }^{3}$ I will not go through all possible variants of the account but rather start by specifying what I take to be a common denominator most explanatory realists should be able to agree on and then discuss some more specific assumptions to prepare the discussion below.

\footnotetext{
2 See Taylor (2018, p. 212); cf. Bader (2021) for an account of stochastic grounding that answers some of Taylor's challenges.

3 Proponents of explanatory realism include Audi (2012a,b), Kim (1994, 1988), Lewis (1986), Ruben (2012), Schaffer (2016), Schnieder (2010), and Skow (2014, 2016).
} 
The term 'explanation' is subject to a familiar process-product ambiguity. ${ }^{4}$ By 'an explanation' one can mean a particular speech act—an act of explaining-or the content of such an act. I will call the latter explanatory information, consisting of an explanans and an explanandum. Explanatory realism is generally viewed as an account of explanatory information. The key idea is that information is explanatory only if the respective explanans is about entities that stand in some suitable relation of determination to an entity the corresponding explanandum is about.

I take explanatory information to consist of propositions, where propositions are construed as relatively fine grained so that there can be different necessarily (even logically) equivalent propositions. ${ }^{5}$ I will call the relation between explanans and explanandum also the explanation relation. We can thus state the general idea of explanatory realism as follows:

(ER) If some propositions $\Gamma$ explain $\phi$ then they provide information about entities that stand in some relation of productive determination to an entity that $\phi$ is about.

A couple of remarks on this are in order.

To begin with, note that the key idea of explanatory realism is sometimes put differently. According to an alternative characterization, realism is the claim that the explanation relation obtains in virtue of the fact that some productive determination relation obtains between entities mentioned in explanans and explanandum. ${ }^{6}$ In this paper, I will not discuss this version of realism, but rather concentrate on (ER). ${ }^{7}$

Next, note that (ER) is merely a necessary condition for the explanation relation to obtain. Explanatory realists typically do not consider it sufficient. Why not? A common reason stems from observations about causation. Plausibly, not all information about the causes of a given event is relevant to explain why the event occurred. If this is the case, a proposition about the causes of a given event might not be relevant to explain why that event occurred. Consider, for instance, cases of switching causation. ${ }^{8}$ Suppose that Suzy throws a rock at a window. Midway through its trajectory the rock is hit by a raindrop sending it to an ever so slightly different trajectory. Suppose that

\footnotetext{
${ }^{4}$ See, e.g., Ruben (2012, p. 6ff) for discussion. Note that in this paper we will be solely concerned with explanation in the sense of explanation why, which is the notion explanatory realists are concerned with. Other uses of 'explanation', e.g. as in 'explaining the meaning of a word', fall outside the scope of our discussion.

5 I will not assume any particular view on propositions. Everything I say below should be compatible with accounts of structured propositions or with an account of propositions in terms of truth-makers along the lines of Fine (2021).

${ }^{6}$ See for instance Kim (1988, p. 226).

7 Note that on plausible assumptions, (ER) entails the alternative characterization. For if (ER) is true for some given $\Gamma$ and $\phi$, it follows that there are some $x x$ such that $\Gamma$ is about the $x x$ and a $y$ such that $\phi$ is about $y$ and $x x R y$ for some productive determination relation $R$. Being an existential generalization, this latter fact is grounded in its instances Fine (2012, p. 59f). In other words, the consequent of (ER) is always grounded in some objects standing in a relation of productive determination. Now, suppose that the consequent of (ER) not only expresses a necessary condition for the explanation relation obtaining, but is also part of a proper analysis of the explanation relation. Since it is often assumed that analyses give rise to grounding relations (see Schnieder 2010), it would then follow that any explanation is partially grounded in the fact that some objects stand in a relation of productive determination.

8 See Paul and Hall (2013, Ch. 5.2) for an overview.
} 
the rock would smash the window on either of the two trajectories. On many popular accounts of causation, the raindrop's hitting the rock will count as part of the causal history of the window's breaking. But it is highly counterintuitive to consider the information that the rock was hit by a raindrop relevant to explain why the window broke. (Of course, if the question were why the window broke in precisely the way it actually broke-i.e. at a certain point of its surface in a certain, specific way- the raindrop's hitting the rock would be explanatorily relevant.) Similar cases have also been discussed with respect to grounding-based explanations. ${ }^{9}$

What further conditions, then, are necessary for explanations to succeed in addition to the one specified in (ER)? What conditions are not fulfilled by propositions about the raindrop's hitting the rock? Different explanatory realists have answered this question in different ways. Some have argued that there are criteria by which one can single out causes that make a difference to the entities mentioned in the explanandum, and that only difference-makers are explanatorily relevant. The raindrop's hitting the rock would be a prime example for a cause that does not make a difference to the occurrence of the explanandum event and that is hence irrelevant. ${ }^{10}$ (Note that those approaches take the property of being a difference-maker to be a non-contextual, objective property.) Others think that successful explanations require the fulfilment of certain epistemic conditions (perhaps additionally to some further, non-epistemic conditions). The raindrop's hitting the rock would thus merely be irrelevant in most epistemic contexts, although it might well be relevant in certain far fetched scenarios. ${ }^{11}$ Finally, there are also philosophers who are sometimes classified as explanatory realists and who would take any proposition about the raindrop's hitting the rock to constitute explanatory information. However, those philosophers stress the importance of distinguishing between explaining well and explaining badly, and would claim that information about the raindrop's hitting the rock can generally merely be used to explain badly why the window broke. ${ }^{12}$ For present purposes, we do not need to adjudicate between these positions. All of them agree on (ER), which Taylor's counterexamples are supposed to prove false. I mentioned them here mainly as a reminder that virtually all participants of the debate take explanations to be more than representations of productive determination relations. This will become important for dealing with the counterexamples.

Two further issues regarding (ER): According to (ER), explanations always involve information about productive determination relations. This raises two questions: (a) what precisely are productive determination relations, and (b) what is meant by 'about'? To answer the first question is a substantive project that I will not pursue in this article. Suffice it to say that the counterexamples we will investigate do not exploit the idea that this notion is unclear or even fundamentally problematic. In general productive determination relations are thought to make or bring about certain events or facts so that they are responsible for their occurrence or obtaining. Uncontroversial examples are causation and grounding, but there might well be other kinds

\footnotetext{
${ }^{9}$ See Schaffer (2012) and Krämer and Roski (2017).

10 See. e.g. Strevens (2004, 2008), Krämer and Roski (2017).

${ }^{11}$ See, e.g. Ruben (2012, p. 194) and cf. Kim (1994, p. 54).

12 Lewis (1986) and Skow (2014).
} 
of productive determination, for instance mereological constitution and other types of building in Bennett's (2017) sense. ${ }^{13}$ For the most part of this article, however, we can do as if grounding and causation were the only productive determination relations. This is not a position that I myself would endorse, but it significantly helps to simplify the discussion. ${ }^{14}$ The question in what sense explanantia and explananda are about the relata of such relations is also not straightforward to answer. ${ }^{15}$ We will see that Taylor's counterexamples exploit precisely this fact. Consequently, in dealing with the counterexamples, I will clarify in what sense explanations are about causes, grounds, effects, and groundees, even if it might appear as though this is not the case. So, while I also won't undertake the project of providing a theory of aboutness suitable for explanatory realism, I will at least highlight some aspects of explanations that such a theory has to account for. In particular, I will discuss the question whether realists have to take every part of a given explanans to provide information about productive determination (Sect. 3), ${ }^{16}$ and I will argue that explanations sometimes provide information about entities through certain pragmatic mechanisms (see Sect. 4.1).

A final remark before I start the discussion. There are two kinds of explanation which are not straightforwardly reconcilable with (ER), but which do play an important role in science. One such kind are explanations of laws and generalizations in terms of other laws. Since these cases do not play any role in Taylor's critique, and since they have been discussed by explanatory realists, I will set them aside. ${ }^{17}$ For the record, I do not think that these explanations give rise to principled problems for explanatory realism anyway. While it might at first seem difficult to reconcile them with the letter of (ER), there are well worked-out realist accounts of such explanations. ${ }^{18}$ And even apart from any particular account, I do not think that such explanations go against the spirit of realism. Laws of nature govern (or perhaps describe, depending on one's view of laws) the causal structure of the world. So, explanations of natural laws in terms of natural laws are in an intuitive sense about the causal structure of the world even if they are not about particular causes or effects (or even types thereof). Another kind of explanation that I will set aside here are explanations of purely mathematical facts in terms of other mathematical facts. There are no causal relations between mathematical facts, and it is an open question whether all inner-mathematical explanations are grounding

\footnotetext{
13 Taylor characterizes productive determination relations as relations that are generative, objective, asymmetric, non-monotonic and explanation-backing (2018, p. 199). These conditions are clearly satisfied for causation and grounding on orthodox accounts of those notions. They are clearly not satisfied for supervenience, entailment, or identity (as neither of those relations is asymmetric). In the literature, authors also often speak about 'determinative or dependency relations' Ruben $(2012,194)$. Taylor generally sticks with 'determination'. Since both the terms 'dependency' and 'determination' are used in a variety of ways that are wider that the sense pertinent in this article, I will stick with 'productive determination'.

14 The position is endorsed by Skow $(2014,2016)$.

15 For some specific domains partial answers have been provided, e.g. by accounts like Schaffer's (2016) that make use of structural equation models following Woodward (2003). For grounding-based explanations cf. Maurin (2019) and Poggiolesi and Genco (2021).

16 That is: whether the 'they' in (ER) is to be read as each or as some.

17 It is indeed not uncommon for explanatory realist to set such explanations aside, see, e.g., Skow (2014, p. 446) and Ruben (2012, p. 4).

18 A view I am partial to is the one advanced by Strevens (2008, Ch. 7).
} 
explanations. ${ }^{19}$ The philosophical investigation into this type kinds of explanation is still in its infancy. To that extent, accounting for them is not a problem that specifically faces realism.

With this out of the way, let us turn to Taylor's counterexamples.

\section{Explanation by analogy}

The first kind of explanation that, according to Taylor, works without representing any causes or grounds are explanations that invoke analogies: analogical explanations as we shall call them. What cases does Taylor have in mind? In her discussion, she hints at various prominent cases from the history of science such as Darwin's analogical use of the notion of selection in evolutionary explanations as well as analogies between the behaviour of billiard balls and molecules in chemical explanations. ${ }^{20}$ Unfortunately, she does not discuss these cases in much detail. I will thus at first concentrate on a toy example that she devotes more attention to and that she seems to consider representative for the whole class: an analogical explanation for why a certain furnace maintains a given temperature during a given temporal interval. Even though I mostly focus on this example, though, my remarks will apply to the class of analogical explanations in general. The example goes as follows:

[(1)] The furnace is analogous to an intentional agent.

[(2)] The furnace's thermostat settings are analogous to an intentional agent's desires.

[(3)] An intentional agent will always aim to promote its desires.

[(4)] By analogy, the furnace will aim to maintain the temperature set for the thermostat, which is $68 \mathrm{~F}$.

[(5)] And that is why the furnace maintains a temperature of 68F. Taylor $((2018$, p. 204); numbers added by me)

Claims (1)-(4) convey the explanans while the indirect question in (5) conveys the explanandum. However, contrary to (ER), Taylor claims, (1)-(4) do not provide any information about causes or grounds of the state that (5) is about.

Explanatory realists could try to dismiss this case by arguing that (1)-(5) do not convey any explanatory information in the first place. And indeed, this particular example might seem a bit far-fetched. (One concern is that the analogy is a bad one. Intentional agents can autonomously change their mind and adjust their intentions, thermostats can't.) Still, whatever one thinks about the case, it would be dialectically unfortunate for explanatory realists to deny analogies any explanatory value outright. Let us, then, accept for the sake of argument that (1)-(5) provide explanatory information and consider why Taylor takes them to provide a counterexample to (ER).

\footnotetext{
19 Lange (2019) and D'Alessandro (2020) argue that this is not the case. Baron et al. (2019, fn. 8) seem at least to consider it a serious possibility. Poggiolesi and Genco (2021) and Betti (2010) are more optimistic. Betti as well as Poggiolesi and Genco are inspired by Bolzano's seminal work on grounding explanations in mathematics. Bolzano himself is another example for an author who took mathematical explanations to be grounding explanations (for discussion, see Roski (2017), Mancosu (1999), and Kitcher (1975)).

20 See Taylor (2018, pp. 204-205).
} 
If we accept that (1)-(5) convey an explanation, it seems natural to insist, against Taylor, that this so because (1)-(4) provide at least some information about causes of the furnace's temperature-even if that information merely tells us that, whatever those causes are, they involve a mechanism that is analogous an intentional agent. Taylor anticipates this objection. In response she claims that "the causal mechanism in the analogy and in the explanandum case are extremely different, which challenges this claim that the explanation explains by giving causal information" (Taylor 2018, 207).

By itself, this is unconvincing. As Taylor admits herself, it is clear that (1)-(5) convey the information that the furnace keeps the temperature because its thermostat is set in a particular way. ${ }^{21}$ Anyone who grasps (1)-(5) should be in a position to obtain this information-quite independently of the information that is additionally conveyed by the analogy. And since there is a causal relation between the setting of the thermostat and the maintenance of the temperature, (ER) is not violated.

So, Taylor's main point must be a different one. She seems to suppose that (i) the content conveyed specifically by the analogy between furnace and intentional agent is explanatory, and that (ii) explanatory realists can only account for this if they take this content to represent features of the causal mechanism in the furnace. Before I answer this challenge on behalf of the realist, let me make one brief observation about a possible motivation for this claim.

It is not clear that realists have to account for the explanatory power of analogies that are part of an explanation in terms of their representational role. Recall that (ER) states that some propositions $\Gamma$ explain $\phi$ only if they are about something that stands in a relation of productive determination to something that $\phi$ is about. Only if one reads 'they' in the statement of this condition distributively, (ER) would require each $\Gamma$ to be about some relatum of a productive determination relation. But explanatory realists are not forced to accept such a reading. Indeed, it seems plausible that different parts of a given explanans can contribute in different ways to the explanatory power of an explanation by fulfilling different roles. And it does not seem to contradict the spirit of explanatory realism if some of these roles do not involve representing productive determination relations. Sometimes information that is given in response to an explanatory request has the function of an epistemic enabler: it helps an audience to recognize that some bit of information about, say, causes that they already possess is explanatory relevant. Epistemic enablers need not represent causes or grounds. ${ }^{22}$ What comes to mind here are, for instance, mathematical equations that are part of a great number of explanations in the natural sciences (e.g. differential equations in physics). Such equations might not represent any causes or grounds by themselves, but they may enable an audience to see that a certain event is in fact causally relevant to another event. All that being said, however, for what follows I will grant Taylor the assumption that, according to realists, the analogy in question is explanatory because it

\footnotetext{
21 This is clearly a somewhat shallow explanation. But such explanations are not entirely unheard of in ordinary contexts: Why does the phone keep playing music for days on end? Because it is plugged in.

22 See Skow (2016, pp. 71-74).
} 
represents features of a causal mechanism in the furnace. In other words, my argument will not hinge on the question of how we read 'they' in (ER). ${ }^{23}$

Would realists be mistaken in claiming that the analogy in the explanation under discussion has a representational role with respect to causes? No. Taylor does not establish that the analogy fails to provide any information about the causal mechanism in the furnace. Indeed, it is difficult to see how this could be the case unless the analogy were a false analogy. But false analogies cannot explain. For instance, one cannot explain why the furnace keeps its temperature by claiming that it works analogously to a randomized process. And in spite of the difference between the two mechanisms in question, the analogy provides information about the causal role of the mechanism operative in the thermostat; roughly along the following lines: just as an agent who sticks to a given aim will adjust their actions depending on the circumstances, the thermostat will adjust the output of the furnace depending on its surroundings to maintain the temperature it is set to. (Note that this does not require causalism about actions: even a non-causal relation may be relevantly analogous to, and thereby help provide information about, causation or causal mechanisms. ${ }^{24}$ ) Taylor's point might be that this information is too unspecific to count as representing causal factors that are responsible for the furnace's maintaining a certain temperature. But it is unclear why explanatory realists should be moved by this point. After all, many causal explanations merely provide highly unspecific information about causal factors. ${ }^{25}$ To explain why a car keeps making a strange sound, it might, in a suitable context, suffice to mention its malfunctioning clutch, or perhaps even simply some malfunction. Just imagine a context where someone is generally unfamiliar with how cars sound normally. To explain why a given window broke, it might in a suitable context suffice to say 'someone threw a rock at it'. Just imagine a context where an insurance agent just needs to know whether the window broke due to an earthquake or due to human involvement. To the extent that Taylor's example is an explanation at all, it seems to differ from such cases at best in degree. What is more, in the right context, the meagre information the analogy provides about the causal mechanism involved in the furnace might just have the right degree of specificity. This could be so if we imagine our audience to consist of small children. Adding further, more specific information about the workings of a thermostat might outstrip the intellectual capacities of this particular audience and prevent the relevant attempt at explaining from being successful.

Note also that the lack of specificity the analogical information suffers from has nothing to do specifically with the fact that it is conveyed by an analogy. It would seem incoherent to accept the original analogical explanation but not an explanation that characterizes the causal role of the mechanism in a similarly unspecific, yet nonanalogical way; for instance, an explanation that simply states that the thermostat adjusts the output of the furnace to the temperature of the environment. Of course,

\footnotetext{
23 Note that I do not want to imply that Taylor wants to defend the strong thesis to the effect that every part of the explanans has to represent entities standing in productive determination relations to the explanandum entity. As far as I can see she does not comment on the issue in her article.

${ }^{24}$ For instance, one may use a visual computer simulation to convey information about, say, the causal goings on in a car engine, even though there is no real causal interaction between parts of the images on the screen that represent parts of the engine.

25 To a certain extent, Strevens's $(2004,2008)$ account of explanation builds on this insight.
} 
such an explanation seems extremely shallow, but there does not seem to be any sense in which the analogical content of Taylor's original explanation provides anything less shallow than this. It would seem equally incoherent to deny that a more elaborate analogy provides information about the causal mechanism in question just because it is an analogy. Think, for instance, of an analogy that compares the mechanism of the furnace to an intricate factory in which various workers carry out various tasks that correspond to features of said mechanism.

I take it that these points generalize: analogies are representational devices-it lies in the nature of analogies to represent features of the world by highlighting salient similarities between different phenomena. ${ }^{26}$ Purported analogies that do not achieve this are-precisely for this reason-false analogies which lack explanatory power altogether. Being representational devices, there is no principled reason why analogies could not be used to represent entities standing in productive determination relations. At any rate, Taylor does not provide us with any general reason to this effect. It might be that she assumes that analogies can represent features of the world only in too unspecific a way to be useful in the context of explanations. But as we have seen from the above discussion it is not clear why this should be the case, and it would, in any case, require a different sort of argument than the one she provides. Note, moreover, that the kinds of analogical explanations Taylor seems to have in mind are explanations of phenomena that are the results of causal processes and hence determined by productive determination relations. Especially in such cases, it seems difficult to see why the respective analogies should fail to represent features of productive determination relations. These remarks apply in particular to the cases of analogical explanations from scientific history that Taylor mentions. Evolution is a causal process. Hence, an analogy between evolutionary mechanisms and an agent who selects among different traits helps explain biological phenomena to the extent that the analogy conveys information about causal mechanisms involved in the process of evolution. Similarly, an analogy between moving balls in a container and the behaviour of gases helps explain chemical phenomena to the extent that the analogy conveys information about the causal processes that the molecules composing those gases undergo.

Let me close this section with some brief observations about the specific purpose of using analogies in explanations. If an analogy is useful to convey information about, say, causes of some event $e$, this is because it enables a given audience to understand why $e$ occurs - typically an audience that only is interested in, or only capable of grasping, certain aspects of $e$ 's causal history. As mentioned earlier, Taylor's case from above seems to be an example for an analogy that might help children to grasp the basic function of a furnace's thermostat ("look, this thermostat-thing here is like a little guy who checks the temperature and diligently takes care that the furnace produces enough heat to keep it at X degrees"). Other analogies might be helpful to make an educated lay audience understand, yet others might even be helpful in strictly scientific contexts where being concise is important, etc. Analogies achieve this by highlighting, relative to different epistemic backgrounds, different aspects of the causal structure that determines a given explanandum event.

26 In this sense, they are akin to models and other idealizations; cf. Potochnik $(2017, \S 2.2 .2)$ 
At one point Taylor seems to suggest that the aims of providing understanding via analogies goes against the spirit of explanatory realism:

One could argue that whatever is going on in these cases it is not explanation, because the primary goal is to help another person to understand some phenomenon, rather than to give information about whatever metaphysically determines that phenomenon. (Taylor 2018, pp. 205-206)

This is a false alternative. The aim of helping to understand does not stand in the way of the requirement articulated by (ER) per se. Of course, adherents of (ER) will insist that every attempt at making someone understand some phenomenon via an explanation involves conveying some information about entities standing in productive determination relations. ${ }^{27}$ However, depending on the audience, different features of those entitites, and different guises of those features, become salient and require the respective explanans be adjusted accordingly. In some cases, analogies are just the right tool for this.

\section{Explanation by rule: conventions and statistics}

Another set of cases that Taylor takes to falsify (ER) are what she calls 'rule-based explanations'. The cases actually fall into two distinct sub-categories. In the first category belong explanations that cite certain statistical rules, the second comprises explanations that cite rules which, roughly speaking, are constitutive of certain social practices. Pertinent examples are rules of games or legal rules. I will begin with discussing this latter category. (A note on terminology: although the two sub-categories differ significantly, I will refer to both of them by the term 'rule-based explanations'. The pertinent category will always be clear from context.)

\subsection{Conventions}

Some explanations refer to rules and regulations. For instance, an explanation for why a football player was sent off might look as follows:

The explanandum is: Player a, who committed a deliberately harmful late tackle, was sent off. The explanans is: The rules of football dictate that any player committing a deliberately harmful late tackle must be sent off. (Taylor 2018, p. 209, emphasis Taylor)

Similarly, an explanation for why someone was sent to prison might cite a specific law that they broke. Taylor takes such explanations to violate (ER). The rules of football do not cause or ground the event of a player's being sent off, and legal rules do not cause or ground people being sent to jail. Rules of football and legal rules are also not to be confused with laws or law-like generalizations. ${ }^{28}$ A rule does not guarantee a given outcome in the same sense in which a law or a generalization does. Rules can

\footnotetext{
27 This is not fully uncontroversial; see Lipton (2009).

${ }^{28}$ See Taylor (2018, p. 210).
} 
be broken and still remain in effect. So, the cases are different from explanations that cite laws or law-like generalizations, which are frequent in scientific contexts.

A natural response to Taylor's cases is to insist that rule-based explanations are ultimately causal in nature and hence do not violate (ER). Whenever a rule is responsible for some player being sent off the field, this is because a referee enforced the rule. Similarly, whenever some legal rule is responsible for sending someone to prison, this is because some judge enforced the legal rule (among other things). So it seems that the rules' explanatory power in Taylor's examples stems from them being involved in the causal histories of the explanandum events by being enforced in one way or another.

Taylor anticipates this objection. She argues that if we construe a rule-based explanation as a kind of causal explanation, we must construe it as a partial causal explanation that does not actually provide any information about causes of the explanandum event. However, she contends, rule-based explanations are not judged partial:

[E]ven if we acknowledge this causal explanation [in terms of the rule and its being enforced, Author], this does not show that the merely rule-based explanation is partial. We can explain the event of a sending off in a variety of different ways, such as appealing to the cause, or appealing to the rules of the game. (Taylor 2018, p. 209)

Her idea seems to be this: If citing a rule in a rule-based explanation does not convey any information about causes, and if adding information about causes of the explanandum event (e.g. a referee's enforcing the rule) does not improve the explanation, we have no reason to judge it partial. And so, she concludes, the original rule-based explanation is an explanation of an event that does not provide any information about causes of the explanandum event. On the assumption that causation is the only relevant productive determination relation in such a case, which seems plausible enough, this conclusion would entail that (ER) is false.

We can agree that there are contexts in which adding causal information indeed does not improve a given rule-based explanation. That is to say, there are contexts where the explanatory request of the audience will be entirely satisfied by a statement of the mere rule. We should deny, however, that citing a rule in an explanation of the kind that Taylor envisages does not convey any information about causes. Taylor seems to assume that the explanatory relevant information conveyed by stating the rule only includes the proposition semantically expressed by it. But this is implausible. Indeed, I submit that we can make sense of rule-based explanations of this kind only if we assume that statements of the rule pragmatically convey information about causes. Hence we should take this information to be part of the full explanation of the event. To see this, imagine a case where some player, $b$, commits a particularly harmful tackle and is sent off by the referee. But now suppose that the referee did not see the tackle, and sent off player $b$ for some other reason. In this case, to claim that player $b$ was sent off because they broke a rule that prohibits harmful tackles is false and hence not explanatory. To be sure, player $b$ did break a rule of this kind. But this is not why they were sent off. Unless the referee actually enforces the relevant rule, citing it has no explanatory power. The legal case is similar. If someone committed a crime that 
violates some law $L$ but is wrongfully charged and sent to prison for a crime they did not commit, it would be false to claim that they were sent to prison because they broke law $L^{29}$

So, even though citing a rule does not semantically express any proposition that is about a cause of the explanandum event, it pragmatically conveys that the rule was enforced by someone. And since enforcing the relevant rule is part of the explanandum event's causal history in the cases under consideration, there is no contradiction to (ER). Incidentally, this also explains why, in many contexts, rule-based explanations are not judged partial. Citing a rule of football to explain why a player was sent off usually conveys the information that the rule was enforced by a referee. Making this latter information explicit is thus, in most contexts, redundant and may even be conversationally inappropriate (due to a violation of the conversational maxim of quantity). This does not stand in the way of the information being part of a full explanation. ${ }^{30}$

Now, Taylor might object that merely pragmatically conveying information about entities standing in productive determination relations does not count as providing information about them. But this would be an odd move. After all, the explanation relation obtains between propositions, and its obtaining should not depend on whether these propositions are semantically expressed or pragmatically conveyed in a given context.

To be clear, there are cases of explanations that cite rules of games or legal rules which do not convey any information about causes. Suppose the question is not why player $a$ was sent off, but rather why it was correct to send off player $a$. In this case, information about causes for why $a$ was sent off would be beside the pointthe information would not just be conversationally inappropriate but explanatorily irrelevant. However, rule-based explanations for why something is correct do not threaten (ER). For, the fact that is correct that $p$ is plausibly grounded by the fact that certain rules dictate that $p$. So, again, we have no contradiction to (ER).

\footnotetext{
${ }^{29}$ A reviewer has raised the question to what extent these considerations generalize. To begin with, my considerations already cover all cases of the kind Taylor seems to have in mind. That is to say, they cover all cases where the behaviour of an agent is explained by a rule that needs to be enforced; i.e. certain legal rules, rules of sports games, etc. Unless such rules are enforced, citing them has no explanatory power. So if statements of the rule are supposed to be explanatory, we have to take them to covey information about the rule's being enforced. But I also think that the considerations generalize even further. For even if the behaviour of an agent is supposed to be explained by a rule that does not need to be enforced, the rule has to be anchored in the behaviour's causal history. For illustration, consider an explanation of an agent's stopping their car in terms of the rule that one has to stop at a red traffic light. Unless we assume that awareness of the rule played some role in the agent's intentional behaviour, we cannot use it to explain said behaviour. For instance, we cannot explain a driver's stopping their car at a red light in terms of a traffic rule if the driver stopped because they ran out of gas.

${ }^{30}$ See Skow (2016, pp. 69-70) for similar observations in a related case. Note that neither Taylor nor I are operating with a particular conception of the distinction between full and partial explanations. Both of us, however, agree that the distinction is a contextual affair; cf. Taylor (2018, p. 209). Whether or not an explanation is full or partial can depend on the background knowledge of the recipients of the explanation and on the particular explanatory request. This is not to say that there is not also an objective sense of 'full explanation'. But this issue seems tangential to my concerns in this paper.
} 


\subsection{Statistics}

The other type of rule-based explanations Taylor considers are explanations that refer to general statistical rules. I will argue that the examples Taylor has in mind are either no genuine explanations or else convey information about causation, which is in line with (ER).

The first example for a statistical rule that Taylor discusses is the Law of Small Numbers which, in her formulation, says:

[W]hen sampling a population for some trait, extreme results (either very high or very low incidence of that trait) are more likely to be found in small samples than in large samples. (Taylor 2018, p. 208)

Taylor now contends that this law can be used to explain why sparsely populated counties exhibit both the lowest and highest incidence of kidney cancer:

[T] he explanandum is the fact that sparsely populated counties exhibit both the lowest and highest incidence of kidney cancer, the explanans is the fact that extreme results are more likely to obtain in smaller samples. (Taylor 2018, p. 208)

Let us grant that the Law of Small Numbers does not convey any information about the causes or grounds of any particular cancer distribution. ${ }^{31}$ In that case, if Taylor's example is a genuine explanation, (ER) is false. However, realists should question the antecedent of this conditional: Taylor's purported explanation is not genuine. Explaining why a particular distribution of cancer incidence in fact prevails requires more than pointing to the mere fact that such a distribution is likely, statistically speaking.

For this reply to have any dialectical force, realists should account for the fact that the information provided by statistical laws seems to resolve a certain type of puzzlement-indeed, a puzzlement that often triggers a why-question. For instance, in the right context, being presented with the information that there appears to be a curious correlation between population density and cancer incidence, one naturally expresses a puzzlement by asking why this is so. I take it that this puzzlement is rooted in presupposing that, in those contexts, the speaker intends to convey that there is some sort of influence between population density and cancer incidence, and consequently wonders about the nature of this influence. Citing the Law of Small Numbers may resolve this puzzlement. It is simply very likely, statistically speaking, that such curious-seeming distributions prevail. But this is merely an artifact of the way the respective samples have been selected and not indicative of any real influence between population density and cancer incidence. The law thus answers the why-question, but not by providing an explanation. By showing that the distribution is not curious at all (statistically speaking), coming to know the law removes a presupposition of the initial request, namely that there is some relation of influence between population density and cancer incidence. (One thereby also learns that it is a bad method of cancer-precaution to move from a sparsely populated high-incidence county to a sparsely populated low-

\footnotetext{
31 Note, however, that there are explanations in biology where similar laws are taken to convey information about causal dispositions; cf. Streven (2008, p. 340).
} 
incidence county, or to a densely populated county.) In a certain sense, we may also say that the law shows that there is nothing to be explained. This interpretation of the case is in line with how Kahnemann, from whom Taylor takes her example, conceives of the case:

The deeper truth is that there is nothing to explain. The incidence of cancer is not truly higher than normal in a county with a small population. [...] [T]he differences between dense and rural counties do not really count as facts: they are what scientists call artifacts, observations that are produced entirely by some aspect of the method of research-in this case, by differences in sample size. (Kahneman 2011, p. 111)

Taylor might reply that removing a puzzlement that triggers a why question with respect to some proposition $\phi$ e o ipso amounts to providing an explanation for $\phi$. But this is surely not true in general. Suppose Sam observes that he has always won at the roulette table when he wore his pink tuxedo. This might seem to be a puzzling coincidence and trigger a corresponding why-question. Sam might, for instance, suspect that the croupier is fond of his tuxedo and exerts some influence on the roulette table Assuming Sam is rational, pointing out to him that there is no such influence, i.e. that the pink tuxedo has nothing to do with his being lucky, should remove his puzzlement. But it clearly doesn't constitute an explanation of his luck. ${ }^{32}$ So, removing a puzzlement that triggered a why-question is not, by itself, providing an explanation. Taylor's purported explanation in terms of the Law of Small Numbers, I submit, is not substantially different. What the law shows is that certain curious-seeming sample distributions are in fact rational to expect, statistically speaking. This way, it can undermine presuppositions about a causal influence between population density and cancer incidence.

Note that I do not want to claim that there are no explanations that invoke statistical rules in Taylor's sense. Such explanations are widespread in sociology and biology. ${ }^{33}$ However, extant accounts of such explanations generally acknowledge that the mere presence of a statistical relationship is insufficient for the presence of an explanatory relationship. In particular, theories of explanation that take into account the use of statistical rules frequently incorporate the assumption that, in explanatory cases, such rules are a guide to causal facts - which is in line with explanatory realism. ${ }^{34}$ (Theories that take mere statistical relevance as sufficient for explanatory relevance are now widely dismissed. ${ }^{35}$ ) This, I take it, is why Kahnemann dismisses the purported explanation of the relation between population density and cancer incidence in terms of the Law of Small Numbers.

\footnotetext{
${ }^{32}$ It might count as a causal explanation in Lewis's (1986) extremely liberal theory of explanatory information since it excludes a certain causal history of Sam's winning, namely that history in which there is a causal influence between tuxedo and roulette outcomes. But that seems to me rather to count against Lewis's theory than against this particular example.

${ }^{33}$ I set aside explanations of non-deterministic phenomena in quantum physics. As Taylor admits, these pose challenges that are independent of explanatory realism, see Taylor (2018, p. 212f).

34 See, e.g., Salmon (1997, p. 476) and Strevens (2008, Chs. 9-11).

${ }^{35}$ See Woodward $(2021, \S 3.4)$ for a brief survey of the problems.
} 
With this in mind, let us have a look at another example of Taylor's for explanations that cite statistical rules: explanations that refer to the Central Limit Theorem. The latter says, very roughly, that the sample mean of a sufficiently large sample approximates a normal distribution. ${ }^{36}$ Taylor now claims that the fact that a particular, sufficiently large sample mean approximates a normal distribution can be explained by the Central Limit Theorem, i.e. by the fact that any sufficiently large sample mean approximates a normal distribution. To pick a particular example: if one rolls two unbiased dice sufficiently often, the mean outcome will approximate 7 . Why is that? Because, thus Taylor's idea, the sample mean of any sufficiently large sample approximates a normal distribution. Does this explanation contradict (ER)? No. For, in this case, realists can plausibly claim that the explanans conveys information about factors that are causally relevant for properties of the series of explanandum events. If the Central Limit Theorem is considered relevant for the explanation of a pattern manifested by the outcomes of a series of throws, this is so because it conveys information about the causal powers of the dice in question, albeit highly unspecific information. Part of this is the information that there is (next to) no causal influence between outcomes of individual throws or between the two dice-otherwise the outcomes could not be represented by independent variables, which is required for the Central Limit Theorem to apply. ${ }^{37}$ In other words, that the Central Limit Theorem can be used to explain a pattern of a particular series of events presupposes that the objects involved in those events have a certain causal profile. Citing the theorem in the context of a successful explanation of a series of actual events thus conveys information about the relata of causation, in line with (ER). ${ }^{38}$

Let me wrap up. I have argued that unless Taylor's examples provide information about causal profiles, they at best show why it is rational to expect a certain outcome without explaining why the outcome in fact occurs. ${ }^{39}$ None of this contradicts (ER).

\section{Explanation by reductio}

A final purported counterexample to (ER) that Taylor invokes are explanations that make use of reductio ad absurdum arguments. In such explanations, Taylor claims,

\footnotetext{
36 See Lyon (2014) for a precise statement and an accessible discussion.

37 Note that it is not at all trivial to apply the Central Limit Theorem for explanatory purposes. See Lyon (2014) for discussion. For a more detailed account on how statistical rules reflect the causal profile of certain entities when used in explanations, see Strevens (2008, p. 370ff).

38 Marc Lange, who discusses cases similar to the ones discussed by Taylor, argues that a statistical explanation "succeeds whether or not any causal relations at all generate the statistical association" (Lange 2017 , p. 193). But this seems wrong. For one, if it were true, it would be difficult to see how one could exclude non-explanatory uses of statistical rules like in Taylor's first example (and in many counterexamples against purely probabilistic theories of explanation). For another, suppose that the dice in the above example were in fact not independent but rather rigged. Suppose further that, by complete accident, they happen to produce a normal distribution in one instance. Surely one cannot use the Central Limit Theorem to explain this particular distribution.

39 I take it that the same is true for similar cases discussed in Lange (2017, pp. 190-196). Of course, statistical rules may also be used in the context of inner-mathematical explanations, in which case they do not convey any information about causes. But these are not the kinds of cases that Taylor has in mind, and they fall outside the scope of this discussion anyway.
} 
we point out that assuming some proposition entails an absurdity and thereby explain why that proposition is true: it is true because it couldn't be false. Taylor contends that there are explanations of this format that violate (ER).

Her main example is an explanation of the fact that there is an absolute zero on the temperature scale, i.e. that there is no temperature of $<0^{\circ}$ Kelvin. ${ }^{40}$ According to Taylor, we can explain this by reductio as follows: ${ }^{41}$

(RK) Suppose there was a temperature of $<0^{\circ}$ Kelvin. By the ideal gas law, such a temperature could only be measured in a gas with negative volume. But there being a gas with negative volume is (physically) impossible. That is why there is no such thing as a temperature of $<0^{\circ}$ Kelvin.

Taylor argues that no fact mentioned in the explanans of (RK) grounds or causes the fact mentioned in the explanandum. ${ }^{42}$

There is a variety of ways explanatory realists can react to this case and to explanations that employ arguments by reductio ad absurdum in general. I will discuss three of those ways that are, to a certain extent, complementary to each other.

To begin with, there is a natural construal of the explanation on which the explanandum is the claim that there is no portion of gas whose temperature deceeds $0^{\circ}$ Kelvin while the explanans are certain physical truths that are incompatible with there being such a portion of gas, including the Ideal Gas Law. Viewed like this, the explanation might satisfy the constraints of being a formal explanation in the sense of Poggiolesi (2018) (given certain assumptions). ${ }^{43}$ Poggiolesi suggests that we should think of the Ideal Gas Law as not strictly speaking belonging to the explanans. Rather, we should think of it as a link in virtue of which certain physical truths explain the fact that there is no portion of gas whose temperature deceeds $0^{\circ}$ Kelvin. ${ }^{44}$ Formally speaking, the law thus appears as a rule rather than a premise. To make this idea a bit more vivid, let us represent the logical form of the explanandum by $\neg \exists x \phi$ and let $P$ be truths jointly incompatible with $\exists x \phi$, given the Ideal Gas Law (IGL). The reductio argument would

\footnotetext{
40 A further example that Taylor mentions is an explanation for why a given star stops collapsing that cites the Pauli Exclusion Principle. The explanation is that, given this principle, it is impossible that a star continues to collapse at a certain point. The example has been much discussed in connection with Lewis's theory of explanation (Lewis 1986, p. 222). However, as Skow (2014, §5) has argued convincingly, the interpretation of the example as a non-causal explanation is deeply problematic, and so I will not discuss the case in this paper.

41 See Taylor (2018, p. 211).

42 See Taylor (2018, p. 211).

43 Those assumptions pertain, first and foremost, to the logical form of explanans and explanandum. In Poggiolesi's system grounds are to be strictly less complex than their groundees (in a certain syntactically defined sense: Poggiolesi (2018, p. 1238)). Whether this is so in the case at hand cannot be answered straightforwardly.

44 This aspect is most explicitly developed in a recent paper with Genco: Poggiolesi (2021, p. 13). We find similar ideas in Schaffer (2017a, p. 3f), Schaffer (2017b, p. 308f), and Kappes (2020). Also Skow's (2016) idea to view laws as second-order reasons why certain truths explain a given explanandum is to a certain extent similarly motivated.
} 
then look roughly like this:

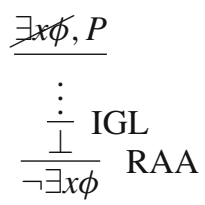

Poggiolesi claims that such formal explanations track grounding relations: their premises (minus the discharged assumption for reductio) represent a ground of the conclusion. They thus show that the negation of a proposition $\phi$ is grounded in certain truths incompatible with $\phi .{ }^{45}$ On Poggiolesi's account, then, that there is no portion of gas with a temperature of $<0$ Kelvin would be grounded in the physical truths that are jointly incompatible with there being such a portion of gas, given the Ideal Gas Law. To transform Poggiolesi's account into a full fledged theory of reductio explanations would require more work. ${ }^{46}$ For present purposes, it suffices to point out that there is a systematically motivated approach to the grounds of negated truths on which Taylor's reductio explanation does not contradict (ER) since it provides information about grounding.

Even if one does not accept Poggiolesi's proposal on explanations by reductio, the explanation can also be quite naturally construed as a kind of causal explanationalbeit a very general one. ${ }^{47}$ In particular, it can be construed as an explanation of a type of event in terms of laws of nature. The explanation tells us that, whenever any portion of gas cools down, it will reach an equilibrium point: a point where it won't further cool down. The Ideal Gas Law thus articulates a constraint on possible causal histories that any particular portion of gas may take. Explanations like this have been investigated by explanatory realists under the heading 'equilibrium explanations'. ${ }^{48}$

\footnotetext{
45 See Poggiolesi (2018, pp. 1232-1233) for the informal idea. Sections 3, 4 of the paper implement this idea in a formal apparatus; cf. also Poggiolesi and Genco (2021). For a general discussion of idea to take the grounds of the negation of a truth $\phi$ to be certain truths incompatible with $\phi$ consider De Rizzo (2019). Note that Poggiolesi develops her account only for the propositional logic of ground. I am taking my above suggestion to be a natural extension of her account. I am grateful to an anonymous reviewer for making me aware of Poggiolesi's account.

46 The account is rigorously developed so far only for logical grounds of negated truths in a propositional language. This means that the theory only allows to obtain grounds of negated truths by deriving a logical contradiction. Since it is not the case that the grounds of any negated truth $\neg \phi$ are logically incompatible with $\phi$, one would have to amend the account to take care of such cases. (Let $\phi$ for instance be the truth that some given apple is not red allover. One might argue that the ground of such a truth is the truth the apple is green allover. But this latter truth is not logically incompatible with the truth that it is red allover.) This problem can arguably be dealt with by employing a more general notion of incompatibility. But working out the details lies beyond the scope of this discussion.

47 Note that these two strategies need not be in competition with each other. On Poggiolesi's view, explanans and explanandum provide information about ground and groundee in a rather direct way, but this is compatible with them also providing information causal information in a more indirect way. Compare a similar case: Jane's parking in the no-parking zone grounds, and explains, her breaking the law. But the information that Jane broke the law by parking in the no parking zone clearly also puts constraints on the causal history of her breaking the law.

48 See, for instance, Skow (2016), Ch. 4, Strevens (2008), p. 266ff, and Woodward (2003), pp. 6-7; cf. also Lange (2017), p. 15.
} 
If we suppose, as seems reasonable enough, that the notion of being about that is employed in (ER) allows to classify explanations that narrowly constrain ${ }^{49}$ the realm of causal histories as being about causes and their effects, then this explanation does not pose any particular problem for realism.

Thus far we have assumed that Taylor's example for an explanation by reductio explains that there is no portion of gas that has a temperature of $<0$ Kelvin in terms of the Ideal Gas Law and other physical truths. But there is another way one might conceive of the explanation, which is perhaps closer to what Taylor has in mind. On this reading, the explanation does not merely show that there is no portion of gas with a temperature of $<0$ Kelvin because of the Ideal Gas Law. It rather shows that things cannot be otherwise: Since the Ideal Gas Law holds with physical necessity, it is physically necessary that there be no portion of gas with a temperature of $<0$ Kelvin, and this latter fact - that there can't be such a thing-on its own explains why there $i$ s none. ${ }^{50}$ Viewed this way, the explanation would be a type of necessitarian explanation, an explanation by modal status. ${ }^{51}$ Such explanations have the following form:

\section{$p$ because $\square p$.}

The role of the reductio argument is to establish the modal status of the explanans. This kind of explanation does indeed raise a challenge for realists. Necessitarian explanations are arguably no grounding explanations. ${ }^{52}$ Are they causal explanations? This is at least unclear. The information provided by the claim that it is physically necessary that there is an absolute zero certainly provides different information than the information that is provided by the Ideal Gas Law and other bits of physical theory in virtue of which this claim holds. While the latter claims refer to properties of gases that are causally relevant to the behaviour of any particular portion of gas, the former is entirely silent about such matters. Hence, it does not seem to be particularly plausible to construe necessitarian explanations as causal. What does this mean for realism?

One possibility is to flat out deny that there are genuine necessitarian explanations. This has been suggested by Schnieder and Kappes. ${ }^{53}$ They argue that why-questions concerning some truth $\phi$ very often come with a presupposition to the effect that

\footnotetext{
49 I am speaking of narrow constraints here, since, on some views of causal explanation, certain facts can causally explain why an event occurred already if they merely exclude a single causal history of that event (Lewis 1986). The constraint on causal histories that the explanation under consideration conveys is much narrower than that.

50 This matches nicely with some claims Taylor makes in discussing the example; for instance: "showing that it would be impossible for things to turn out any other way is to give a good reason for why the temperature scale has a lowest point" (Taylor 2018, p. 212, emph. Taylor). "The explanandum is that there is a lowest point, absolute zero, on the temperature scale. The explanation is that if there were no absolute zero then an impossible thing would happen, because a gas would have a negative volume. The impossible thing cannot happen, and so there must be absolute zero" (Taylor 2018, p. 211). Note that on this construal of the explanation, we do not need not make any assumptions about the logical form of the explanandum (such as it being a negated existential claim).

51 See Glazier (2017), $\$ 3$ for an account of such explanations.

52 See Glazier (2017), pp. 1418-1419.

53 See Kappes (2020), Kappes and Schnieder (2016), and cf. also Skow (2016, Ch. 5).
} 
$\phi$ could also be false. Pointing out that $\phi$ is physically necessary undermines the presupposition of this question. This might constitute a satisfactory answer to the why-question without being an explanation. (We have observed a similar phenomenon above in \$4.2.) Kappes and Schnieder further argue that knowing that $\phi$ is necessary may further epistemic goals that are often associated with explanations such as expectability. ${ }^{54} \mathrm{I}$ am very sympathetic to this reply. At least as far as my intuitions are concerned, responding to a genuine explanation-seeking why question with respect to $\phi$ by pointing out that $\phi$ is necessary is unsatisfactory. (In a similar way in which it is unsatisfactory to answer the question 'Why is Socrates mortal?' by 'Because all human beings are'. ${ }^{55}$ ) Such an answer might well strengthen one's confidence in the truth of the explanandum. But it naturally invites further why-questions: questions regarding the source of $\phi$ 's necessity (or the source of humans' being mortal). In the case at hand, a more satisfactory answer would cite the Ideal Gas Law, and perhaps point out that it is a law of nature, which would take us back to the causal interpretation of the explanation sketched above.

Another possibility to accommodate necessitarian explanations is to take a cue from Skow (2016) and construe them not as explanations of the explanandum in question, but rather as second-order explanations; that is, as explanations for why a certain explicitly or contextually given claim explains why there is no absolute zero. A bit more concretely, there are contexts in which an interlocutor may ask why there is an absolute zero even if they are familiar with the explanation in terms of the Ideal Gas Law. To this question, it seems legitimate to answer 'because it must be so'. Such an answer not only highlights the modal status of explanans and explanandum of the original (firstorder) explanation, it also points to the nature of the connection between explanans and explanandum. In particular, it shows that the explanandum is a result of the causal structure of the world that is dictated by the laws of physics. Now, since secondorder explanations that help explain why something is an explanation can naturally be construed as grounding explanations, we again do not have any contradiction to (ER).

Where do we stand? I have discussed two ways to construe Taylor's example for an explanation by reductio ad absurdum. On one construal, the explanation explains the nonexistence of any portion of gas with a temperature of $<0$ Kelvin in terms of the Ideal Gas Law and other physical truths. Such an explanation can be viewed as a grounding explanation in line with Poggiolesi (2018) or as a type-level causal explanation-both of which is consistent with (ER). Alternatively, the case that Taylor discusses can be construed as conveying a necessitarian explanation. While this kind of explanation does raise a challenge to (ER), I have argued that it is a challenge that can be answered.

\section{Conclusion}

Taylor has argued that explanatory realism is false since (ER), a necessary condition characteristic of the view, is false. We have seen that realists need not be worried by the cases she adduces in support of this claim. On a reasonable construal of 'about',

\footnotetext{
54 See Kappes (2020), §4 and Kappes and Schnieder (2016), pp. 557-558.

55 See Dretske (1977), p. 262 and Roski (2018), p. 1998f for elaboration of this intuition.
} 
all of those examples are about relata of productive determination relations or are not cases of genuine explanation. Analogical explanations represent contextually salient aspects of causes or causal histories. Statements of rules of games or legal rules that do not semantically express propositions that are about causes nevertheless convey such propositions in contexts where they are used to explain the occurrence of certain events. Statistical rules, realists can claim, do not have explanatory power by themselves unless they are interpreted causally. Finally, we have seen a variety of ways in which realists can account for reductio explanations in their framework. Explanatory realism stands undefeated.

Acknowledgements For comments on previous versions of this article, I'd like to thank Singa Behrens, Alexander Dinges, Yannic Kappes, Benjamin Kiesewetter, Martin Glazier, Patricia Rich, Stefan Rinner, Julio De Rizzo and Moritz Schulz. I am especially grateful to Stephan Krämer for several very helpful comments. I'd also like to thank two anonymous referees for this journal for their helpful comments. Work on this paper was funded by the Deutsche Forschungsgemeinschaft through an Emmy Noether grant for the project Relevance (Grant KR 4516/2-1). I am very grateful for this support.

Funding Open Access funding enabled and organized by Projekt DEAL.

Open Access This article is licensed under a Creative Commons Attribution 4.0 International License, which permits use, sharing, adaptation, distribution and reproduction in any medium or format, as long as you give appropriate credit to the original author(s) and the source, provide a link to the Creative Commons licence, and indicate if changes were made. The images or other third party material in this article are included in the article's Creative Commons licence, unless indicated otherwise in a credit line to the material. If material is not included in the article's Creative Commons licence and your intended use is not permitted by statutory regulation or exceeds the permitted use, you will need to obtain permission directly from the copyright holder. To view a copy of this licence, visit http://creativecommons.org/licenses/by/4.0/.

\section{References}

Audi, P. (2012a). A clarification and defense of the notion of grounding. In F. Correia \& B. Schnieder (Eds.), Metaphysical grounding (pp. 101-121). Cambridge: Cambridge University Press.

Audi, P. (2012b). Grounding: Toward a theory of the in-virtue-of relation. The Journal of Philosophy, $109(12), 685-711$.

Bader, R. (2021). The fundamental and the brute. Philosophical Studies, 178, 1121-1142.

Baron, S., Colyvan, M., \& Ripley, D. (2019). A counterfactual approach to explanation in mathematics. Philosophia Mathematica Advance Access, 28, 1-34.

Bennett, K. (2017). Making things up. Oxford and New York: Oxford University Press.

Betti, A. (2010). Explanation in metaphysics and Bolzano's theory of ground and consequence. Logique et Analyse, 211(53), 281-316.

D'Alessandro, W. (2020). Viewing-as explanations and ontic dependence. Philosophical Studies, 177(3), $769-792$.

De Rizzo, J. (2019). Reasons why not. On the positive grounds of negative truths. Berlin: J. B. Metzler.

Dretske, F. (1977). Laws of nature. Philosophy of Science, 44(2), 248-268.

Fine, K. (2012). Laws of nature. In F. Correia \& B. Schnieder (Eds.), Metaphysical grounding (pp. 37-80). Cambridge: Cambridge University Press.

Fine, K. (2021). A theory of truthmaker content. Journal of Philosophical Logic, 46(6), 625-674.

Glazier, M. (2017). The difference between epistemic and metaphysical necessity. Synthese, 198(Suppl 6), 1409-1424.

Kahneman, D. (2011). Thinking, fast and slow. Oxford and New York: Oxford University Press.

Kappes, Y. (2020). Explanation by status as empty-base explanation. Synthese Online First, 1-21.

Kappes, Y., \& Schnieder, B. (2016). Anything at all: The deepest and the shallowest question. Philosophisches Jahrbuch, 123(2), 543-565. 
Kim, J. (1988). Explanatory realism, causal realism, and explanatory exclusion. Midwest Studies in Philosophy, 12(1), 225-239.

Kim, J. (1994). Explanatory knowledge and metaphysical dependence. Philosophical Issues, 5, 51-69.

Kitcher, P. (1975). Bolzano's ideal of algebraic analysis. Studies in History and Philosophy of Science, 6(3), 229-269.

Krämer, S., \& Roski, S. (2017). Difference-making grounds. Philosophical Studies, 174(5), 1191-1215.

Lange, M. (2017). Because without cause. New York: Oxford University Press.

Lange, M. (2019). Ground and explanation in mathematics. Philosophers' Imprint, 19(33), 1-18.

Lewis, D. (1986). Causal explanation. In D. Lewis (Ed.), Philosophical papers (Vol. II, pp. 214-240). Oxford and New York: Oxford University Press.

Lipton, P. (2009). Understanding without explanation. In H. W. de Regt, S. Leonelli, \& K. Eigner (Eds.), Scientific understanding: Philosophical perspectives (pp. 43-63). Pittsburgh: University of Pittsburgh Press.

Lyon, A. (2014). Why are normal distributions normal? British Journal for the Philosophy of Science, 65(3), 621-649.

Mancosu, P. (1999). Bolzano and Cournot on mathematical explanation. Revue d'Histoire des Sciences, 52, $429-455$.

Maurin, A.-S. (2019). Grounding and metaphysical explanation: It's complicated. Philosophical Studies, 176, 1573-1594.

Paul, L., \& Hall, N. (2013). Causation: A users guide. Oxford: Oxford University Press.

Poggiolesi, F. (2018). On constructing a logic for the notion of complete and immediate formal grounding. Synthese, 195(3), 1231-1254.

Poggiolesi, F., \& Genco, F. (2021). Conceptual (and hence mathematical) explanation, conceptual grounding and proof. Erkenntnis Online First.

Potochnik, A. (2017). Idealization and the aims of science. Chicago: Chicago University Press.

Roski, S. (2017). Bolzano's conception of grounding. Frankfurt am Main: Vittorio Klostermann.

Roski, S. (2018). Grounding and the explanatory role of generalizations. Philosophical Studies, 175(8), 1985-2003.

Ruben, D.-H. (2012). Explaining explanation (updated and extended) (2nd ed.). Boulder: Paradigm Publishers.

Salmon, W. (1997). Causality and explanation: A reply to two critiques. Philosophy of Science, 64(3), $461-477$.

Schaffer, J. (2012). Grounding, transitivity and contrastivity. In F. Correia \& B. Schnieder (Eds.), Metaphysical grounding (pp. 122-138). Cambridge: Cambridge University Press.

Schaffer, J. (2016). Grounding in the image of causation. Philosophical Studies, 173(1), 49-100.

Schaffer, J. (2017a). The ground between the gaps. Philosophers' Imprint, 17(11), 1-26.

Schaffer, J. (2017b). Laws for metaphysical explanation. Philosophical Issues, 27, 302-321.

Schnieder, B. (2010). A Puzzle about 'Because'. Logique et Analyse, 53(211), 317-343.

Skow, B. (2014). Are there non-causal explanations (of particular events)? British Journal for the Philosophy of Science, 65(3), 445-467.

Skow, B. (2016). Reasons why. Oxford and New York: Oxford University Press.

Strevens, M. (2004). The causal and unification approaches to explanation unified-causally. Nô̂s, 38(1), 154-176.

Strevens, M. (2008). Depth. Cambridge, MA: Harvard University Press.

Taylor, E. (2018). Against explanatory realism. Philosophical Studies, 175(1), 197-219.

Woodward, J. (2003). Making things happen. Oxford and New York: Oxford University Press.

Woodward, J. (2021). Scientific explanation. In E. N. Zalta (Ed.), The Stanford encyclopedia of philosophy (Spring 2021 ed.). Metaphysics Research Lab, Stanford University.

Publisher's Note Springer Nature remains neutral with regard to jurisdictional claims in published maps and institutional affiliations. 